\title{
ENFOQUES TEÓRICOS EN EL ESTUDIO DE LA ADMINISTRACIÓN Y REFORMA A LA JUSTICIA EN COLOMBIA
}

\section{THEORIC FOCUS IN THE STUDY OF THE ADMINISTRATION AND REFORM OF JUSTICE IN COLOMBIA}

\author{
ROSEMBERT ARIZA SANTAMARÍA \\ Docente Investigador Universidad Santo Tomás y Universidad Nacional de Colombia \\ GINNA MARCELA RIVERA RODRÍGUEZ \\ Estudiante Maestría en Estudios Políticos IEPRI Universidad Nacional de Colombia
}

Fecha de recepción: 29 de abril de 2010.

Fecha de aprobación: 20 de mayo de 2010.

\begin{abstract}
Resumen:
Este texto busca delinear sintéticamente las gruesas líneas teóricas que enmarcan los estudios revisados de la literatura sobre administración de justicia y reforma judicial en Colombia en las dos últimas décadas.
\end{abstract}

\footnotetext{
Abstrac:

This text looks for synthetically to delineate the heavy theoretical lines that frame the studies reviewed of Literature on administration of justice and judicial reform in Colombia in the two last decades.
} 


\section{Palabras clave:}

Reforma Judicial, Instrumentalismo, institucionalismo, neoconstitucionalismo, neoliberalismo

\section{Key words:}

The Judicial Reformation, Instrumentalismo, institucionalismo, neoconstitucionalismo, neoliberalism.

\section{Identificación del Artículo:}

Reflexión académica.

Sabemos de la importancia que tiene para los investigadores explicar y comprender asuntos como el origen, contenido, ejes conceptuales, efectos y resultados de las reformas, pero también de lo fundamental que ello resulta para presentes y futuros procesos de discusión y decisión de política pública (en todo su ciclo) en el tema.

Al respecto, valga aclarar que la lectura interpretativa de la que se dará cuenta en las próximas líneas, combinará un doble ejercicio de rastreo que comprende: Una breve presentación de los postulados teóricos generales y los elementos 


\section{REVISTA VIRTUAL VIA INVENIENDI ET IUDICANDI \\ "CAMINO DEL HALLAZGO Y DEL JUICIO" \\ http://viei.usta.edu.co/ E-MAIL: revistainveniendi@usantotomas.edu.co}

conceptuales que dan contenido a cada corriente teórica según lo expuesto por los autores revisados, así como la sistematización, en clave de síntesis, de las propuestas planteadas desde las corrientes teóricas frente al tema de reforma judicial.

Los diversos estudios revisados han pretendido responder a interrogantes sobre el rol de las instituciones en la administración de justicia, la relación de esta última con el desarrollo económico de los países, el papel de los actores que se han hecho y pueden hacerse partícipes del proceso de cambio, entre otras tantas. Sin embargo, tal y como se evidenciará a continuación las respuestas enunciadas frente a tales interrogantes han sido múltiples y variadas, razón por la cual constituyen el hilo conductor de identificación de las distintas corrientes, como sigue:

García, Rodríguez y Uprimny (2006:18) afirman que desde el punto de vista teórico, las posiciones sobre las instituciones en el ámbito de la justicia pueden dividirse en instrumentalistas y constructivistas. La primera orientación, que domina en la ciencia económica, concibe la posibilidad de utilizar las instituciones como instrumento de intervención y acción social, es decir, "tiende a ver las instituciones como tareas técnicas encaminadas a moldear la práctica jurídica, económica o política -por ejemplo, para promover el desarrollo económico o el 


\section{REVISTA VIRTUAL VIA INVENIENDI ET IUDICANDI \\ "CAMINO DEL HALLAZGO Y DEL JUICIO" \\ http://viei.usta.edu.co/ E-MAIL: revistainveniendi@usantotomas.edu.co}

Estado de derecho-, independientemente del contexto social en el que dichas instituciones están llamadas a operar”. (García, et al, 2006:19)

Algunas respuestas que esta postura da a preguntas como las mencionadas anteriormente, son: Según Becker (1976), citado por los autores (García, et al, 2006:19), los actores sociales son vistos como agentes racionales que se comportan al modo de quienes participan en el mercado. A su vez, las condiciones de estabilidad institucional, resultan fundamentales por un lado, para garantizar derechos, especialmente, los de propiedad, y por el otro para asegurar la eficiencia del mercado, necesaria para generar desarrollo en las naciones.

Respecto a la segunda orientación teórica, en contraposición a la primera, autores de diversas ciencias sociales han formulado una aproximación teórica que para Berger y Luckman (1966), citados por los autores (García, et al, 2006:20) es:

Una corriente sociológica que, en oposición al instrumentalismo, considera que los sujetos y las instituciones son realidades que resultan del contexto social y cultural en el que operan y que, por lo tanto, no pueden ser manipuladas o intervenidas con facilidad "desde afuera" mediante procedimientos de ingeniería social como los propuestos por los enfoques instrumentalistas. 


\section{REVISTA VIRTUAL VIA INVENIENDI ET IUDICANDI \\ "CAMINO DEL HALLAZGO Y DEL JUICIO" \\ http://viei.usta.edu.co/ E-MAIL: revistainveniendi@usantotomas.edu.co}

La interpretación propuesta desde esta postura para el análisis de las instituciones, permite verlas en una estrecha conexión con las prácticas sociales, a partir de lo cual se infiere que las reformas institucionales "lejos de ser problemas técnicos independientes del contexto en el que operan, tienen orígenes y efectos profundamente dependientes de dicho contexto". (García, et al, 2006:20)

Sumadas a estas dos primeras orientaciones desde el punto de vista teórico, García, Rodríguez y Uprimny (2006:20-21), sostienen que en el conjunto de respuestas a los interrogantes planteados sobre la justicia, es posible reconocer un punto de vista valorativo o político, porque el que tales respuestas pueden ser agrupadas en visiones libertarias y en perspectivas igualitarias. En el primer grupo, existe una concepción sobre el mercado como "instrumento fundamental de coordinación social" y la creencia en que las instituciones en general, y el derecho en particular, deben permitir que éste cumpla con dicha función, sin obstáculos. En el segundo, se defiende la idea de que el mercado no puede ser un instrumento equitativo de regulación social sin que previamente se hayan garantizado ciertas condiciones mínimas de igualdad entre los actores que de él participan. Por ello esta visión defiende un rol más protagónico del Estado en la vida social y un concepto amplio sobre las instituciones sociales que no las reduzca a ser garantes del orden público, la propiedad privada y la seguridad de los contratos, sino que sean fuertes para incluir también la protección de otras libertades civiles, como el acceso igualitario a la justicia, a los derechos sociales, económicos y culturales. 


\section{REVISTA VIRTUAL VIA INVENIENIDI ET IUDICANDI \\ "CAMINO DEL HALLAZGO Y DEL JUICIO"}

http://viei.usta.edu.co/ E-MAIL: revistainveniendi@usantotomas.edu.co

Al cruzar los dos ejes mencionados, el teórico y el valorativo, los autores (García, et al, 2006: 23) plantean el siguiente mapa que refleja cuatro aproximaciones distintas a las instituciones, a la justicia y a la reforma judicial:

\begin{tabular}{|c|c|c|c|}
\hline \multicolumn{2}{|c|}{ Dimensión valorativa } & & \multirow{4}{*}{ 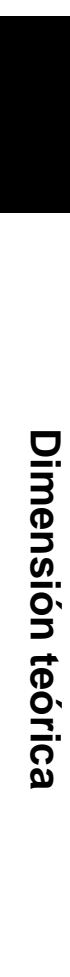 } \\
\hline Liberal individualista & Igualitaria & & \\
\hline $\begin{array}{l}\text { Neoinstitucionalismo; } \\
\text { movimiento de derecho y } \\
\text { economía } \quad \text { (p. ej. } \\
\text { Programas recientes de } \\
\text { reforma judicial) }\end{array}$ & $\begin{array}{l}\text { Desarrollismo (p. ej. } \\
\text { "primera ola" de } \\
\text { programas de derecho y } \\
\text { desarrollo) }\end{array}$ & 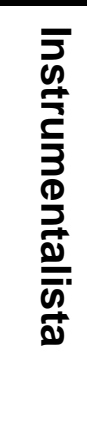 & \\
\hline $\begin{array}{l}\text { Posmodernismo (p. ej. } \\
\text { Estudios de conciencia } \\
\text { jurídica) }\end{array}$ & $\begin{array}{l}\text { Institucionalismo social } \\
\text { (p. ej. Constitucionalismo } \\
\text { social) }\end{array}$ & 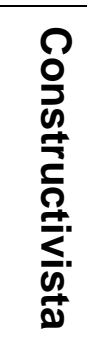 & \\
\hline
\end{tabular}

La combinación de las opciones dentro de las dos dimensiones (valorativa y teórica) da como resultado la identificación de cuatro posturas, tal y como se presenta en la tabla, sin embargo, los autores hacen énfasis en las siguientes:

1. Una visión instrumentalista de las instituciones y una preferencia valorativa por los derechos de libertad (propiedad y libertad comercial) constituye el núcleo del neoinstitucionalismo económico. 


\section{REVISTA VIRTUAL VIA INVENIENDI ET IUDICANDI \\ "CAMINO DEL HALLAZGO Y DEL JUICIO"}

http://viei.usta.edu.co/ E-MAIL: revistainveniendi@usantotomas.edu.co

2. Una visión constructivista sobre las instituciones y una opción valorativa igualitaria sobre los derechos y la inclusión social conforman el foco de interés del institucionalismo social.

Sobre ésta último, como propuesta teórica y valorativa de García, Rodríguez y Uprimny es posible mencionar que dentro de su núcleo de propuestas se considera que una agenda de reforma equitativa e incluyente debe dar un lugar central al diseño institucional, o si se quiere, que es posible lograr cambios sociales a través de las transformaciones institucionales. Por ello "se trata de tomar en serio a las instituciones y de hacerlo a partir de una perspectiva de filosofía moral y de políticas públicas que le den tanta importancia a la libertad como a la igualdad, a la eficacia como a la inclusión social". (García, et al, 2006: 25-26)

Por su parte, el mismo Mauricio García (2006) en su reflexión sobre justicia, mercado y democracia presenta los postulados fundamentales del movimiento de derecho y economía y del neoinstitucionalismo económico mencionados con anterioridad, como dos corrientes teóricas económicas que interesan señalar, dada su importancia para el debate académico y el debate público. De lo mencionado por el autor, se destacan los siguientes apartes: 


\section{REVISTA VIRTUAL VIA INVENIENDI ET IUDICANDI \\ "CAMINO DEL HALLAZGO Y DEL JUICIO" \\ http://viei.usta.edu.co/ E-MAIL: revistainveniendi@usantotomas.edu.co}

\section{Movimiento de derecho y economía (law and economics)}

Su origen data de los años sesenta, a partir del trabajo Ronald Coase (1960), quien critica

El principio económico clásico, según el cual las externalidades del sistema económico -que resultan cuando la decisión de una persona de producir o consumir afecta a otra- deben ser remediadas mediante intervenciones del Estado a través del derecho, es decir, mediante regulaciones. De acuerdo con esta visión clásica, para prevenir la ineficiencia en la adjudicación de recursos, el sistema jurídico debe hacer responsables a quienes afectan a otros cuando intervienen en el mercado. Con dicha intervención, las externalidades indeseables resultan internalizadas por los responsables de los costos adicionales del mercado. En contra de este principio clásico, Coase sostuvo, que bajo condiciones de costos de transacción mínimos y cuando los derechos de propiedad están claramente delimitados, la intervención del derecho no tiene consecuencias en la adjudicación de recursos; por lo tanto, la mejor solución viene de la negociación entre los individuos involucrados. (García, 2006: 61)

De ello se entiende, que los individuos particulares tienen mejores incentivos que el Estado para encontrar soluciones eficientes al problema de las externalidades. Este teorema de Coase permitió que muchos profesionales del derecho encontrarán el sustento teórico para rechazar las políticas intervencionistas del 


\section{REVISTA VIRTUAL VIA INVENIENIDI ET IUDICANDI \\ "CAMINO DEL HALLAZGO Y DEL JUICIO"}

http://viei.usta.edu.co/E-MAIL: revistainveniendi@usantotomas.edu.co

Estado y favorecer la desregulación de la economía. Todo ello promovió un gran movimiento conservador "que trajo la idea de que la economía podía proporcionar el bagaje teórico necesario para crear una teoría del derecho realmente científica, neutral y apolítica" (García, 2006: 62)

\section{Neoinstitucionalismo económico}

Esta corriente sostiene que existe una influencia recíproca entre comportamiento económico y ambiente institucional, razón por la cual sus postulados sugieren una crítica fuerte a la concepción liberal de un mercado eficaz y autosuficiente, insistiendo en que el mercado no se hace solo sino que requiere de instituciones fuertes que lo hagan posible. Para desarrollar los aportes de esta corriente García (2006: 71-72) presenta una síntesis de los trabajos de Douglass North, a quien se considera el vocero más reputado de la misma, cuyos principales aspectos se mencionan a continuación:

El neoinstitucionalismo sostiene que la cooperación entre los actores económicos no siempre es predecible, debido a que la motivación de los actores es compleja e inestable y a que los intercambios conllevan costos elevados de transacción que sólo pueden atenuarse a través de la introducción de instituciones. Como puede verse, North rechaza el postulado del actor racional que defiende el movimiento de derecho y economía. 


\section{REVISTA VIRTUAL VIA INVENIENDI ET IUDICANDI \\ "CAMINO DEL HALLAZGO Y DEL JUICIO" \\ http://viei.usta.edu.co/ E-MAIL: revistainveniendi@usantotomas.edu.co}

Respecto al papel de las instituciones desde esta postura, North, citado por García (2006: 72) plantea que estas "reducen las incertidumbres propias de la acción humana. Ellas proporcionan la estructura de intercambio que (junto con la tecnología) determina el costo de la transacción y el costo de la transformación". Tal capacidad de acción de las instituciones resulta de su posibilidad para imponer normas de comportamiento a los participantes. "Estas normas, según North, puede ser formales -por ejemplo las leyes, las constituciones, los reglamentos, etc.- o informales, que consisten en filtros culturales o ideológicos a partir de los cuales interpretamos los hechos e incluso las mismas normas formales". (Ibíd.)

Como complemento de lo expuesto por García, resulta importante, referenciar el tratamiento de esta corriente por parte de otros autores como los que siguen:

Rodríguez y Uprimny (2006:124) afirman que esta corriente pretende:

Corregir ciertas insuficiencias de la economía neoclásica, y por ello insisten en que el mercado no se desenvuelve en el vacío, sino requiere de instituciones que lo hagan posible. La tesis básica es que mientras más elevados sean los costos de transacción, menos desarrollados y eficientes serán los mercados, pues más difícil será poder realizar un intercambio y menores serán las posibilidades de crecimiento. 


\section{REVISTA VIRTUAL VIA INVENIENDI ET IUDICANDI \\ "CAMINO DEL HALLAZGO Y DEL JUICIO" \\ http://viei.usta.edu.co/ E-MAIL: revistainveniendi@usantotomas.edu.co}

Por su parte García Chourio (2003: 117) sintetiza los postulados de esta corriente a partir de los trabajos de Vieux y Petras (1996), los llamados estatistas neoliberales, afirmando que el neoinstitucionalismo es:

Aquel en donde todo actor, no importando el origen de su base -nacional o internacional, privada, pública o neogubernamental-, se convierte de manera eficiente en parte del sistema de mercado si es disciplinado por las instituciones y los procedimientos de este sistema, o sea, si está de acuerdo con las reglas que plantea el sistema, lo cual implica un espacio estatal para construir el compromiso y la identificación con dichas reglas.

García Chourio siguiendo la posición moderada, como él mismo la llama, de autores como March y Olsen $(1993,13)$ comparte la idea que admite en el neoinstitucionalismo ciertos elementos de la teoría de la elección racional, debido a que reconoce a las instituciones como un "juego de herramientas" del que los actores seleccionan racionalmente distintas piezas para llevar a cabo sus acciones. Por último el autor rescata otra de las premisas de la corriente neoinstitucionalista a partir de la cual se admite relativa autonomía al Estado para el desempeño de instituciones formales e informales, que como tal tienen gran importancia para el desarrollo económico dada su posibilidad de proporcionar previsibilidad, sobre y para los actores. (García Chourio: 2003: 118) 


\section{REVISTA VIRTUAL VIA INVENIENDI ET IUDICANDI \\ "CAMINO DEL HALLAZGO Y DEL JUICIO" \\ http://viei.usta.edu.co/ E-MAIL: revistainveniendi@usantotomas.edu.co}

La siguiente fuente teórica, identificada en la literatura revisada, está compuesta por los criterios orientadores de la teoría de la elección racional, ejemplo de ello es el trabajo de Sonia Fleury (2003: 85-86) quien sostiene que:

La teoría de la elección racional presupone que las personas eligen el curso de acción que prefieren o creen es la mejor. Las decisiones racionales están basadas en cálculos de costo/beneficio individuales, y en las decisiones interdependientes, dominio conocido como la teoría de los juegos. La contribución de la teoría económica neoclásica de elección racional a la comprensión de los microfundamentos del comportamiento es reconocida por diferentes corrientes (Elster, 1989, Roemer, 1989), aunque se critica la predominancia del individualismo en aquella interpretación, que conlleva a dos confusiones: identificar preferencias con elecciones y elección con bienestar. Las personas no siempre escogen lo que prefieren, ni tampoco eligen o prefieren lo que les conduce al bienestar (Roemer, 1989).

Esta misma autora (Fleury, 2003: 86-87) permite esbozar algunos aspectos definitorios de otra de las corrientes teóricas encontradas, la teoría de agencia:

La contribución de la teoría de la elección racional aplicada a la reforma del Estado es conocida como la teoría de la agencia. Manteniendo los mismos principios utilitarios clásicos acerca de las motivaciones individuales en la toma de decisiones racionales que maximicen sus intereses, la actualización 


\section{REVISTA VIRTUAL VIA INVENIENDI ET IUDICANDI \\ "CAMINO DEL HALLAZGO Y DEL JUICIO" \\ http://viei.usta.edu.co/E-MAIL: revistainveniendi@usantotomas.edu.co}

se aplica a agentes económicos que actúan en un mercado imperfecto, con grados diferenciados de acceso a las informaciones necesarias para la toma de decisiones. Según Przeworsky (1998), si se parte del supuesto de la imperfección del mercado, el problema que se presenta consiste en identificar las instituciones específicas que podrían inducir a los actores individuales - sean ellos agentes económicos, políticos o burócratas- a comportarse de manera que beneficie a la colectividad. El problema de la agencia empieza porque los gerentes estarán frecuentemente tentados a actuar en favor de sus propios intereses, y no en favor de los intereses de la organización que les contrata. La teoría de la agencia ofrece dos respuestas para el alineamiento de los intereses. La primera es aumentar el flujo de informaciones del principal a través de indicadores de desempeño, aumentar la rendición de cuentas financieras y especificar claramente los objetivos. La segunda es contratar en el mercado, puesto que la competición actúa como disciplina de los agentes.

Ahora bien, respecto a la sistematización de lo que las corrientes teóricas proponen a la reforma judicial específicamente, hemos construido la siguiente matriz de síntesis de lo sustentado por los autores: 


\begin{tabular}{|l|l|}
\hline \multicolumn{1}{|c|}{$\begin{array}{c}\text { CORRIENTES } \\
\text { TEÓRICAS }\end{array}$} & \multicolumn{1}{|c|}{ REFORMA JUDICIAL: CONTENIDOS, ACCIONES Y } \\
PROPUESTAS
\end{tabular}




\begin{tabular}{|c|c|}
\hline $\begin{array}{l}\text { CORRIENTES } \\
\text { TEÓRICAS }\end{array}$ & $\begin{array}{l}\text { REFORMA JUDICIAL: CONTENIDOS, ACCIONES Y } \\
\text { PROPUESTAS }\end{array}$ \\
\hline $\begin{array}{l}\text { Institucionalismo } \\
\text { social }\end{array}$ & $\begin{array}{l}\text { constructivista de las políticas públicas, que resalta la } \\
\text { importancia de consultar el contexto social y los } \\
\text { individuos afectados por las reformas propuestas. La } \\
\text { propuesta contiene los siguientes postulados: } \\
\text { 1. La limitada inclusión social por medio del mercado } \\
\text { se debe complementar con una fuerte inclusión por } \\
\text { medio de la participación democrática y la defensa } \\
\text { de los derechos } \\
\text { 2. Las reformas exitosas son aquellas que } \\
\text { incrementan la independencia judicial- y por lo } \\
\text { tanto, modifican el contexto político en el que } \\
\text { operan- y promueven el acceso efectivo y } \\
\text { equitativo a la justicia-, y con ello, atienden las } \\
\text { necesidades del contexto social de ésta. } \\
\text { 3. El balance entre la protección jurídica eficaz de los } \\
\text { derechos y la limitación del poder de los jueces, } \\
\text { requiere una teoría que defienda la autonomía de } \\
\text { los jueces, y promueva la adopción de criterios de } \\
\text { decisión transparentes y responsables. (García, et } \\
\text { al, 2006: } 25-28 \text { ) }\end{array}$ \\
\hline $\begin{array}{l}\text { Movimiento de } \\
\text { derecho y economía }\end{array}$ & $\begin{array}{l}\text { Las reformas deben ir encaminadas a mejorar la } \\
\text { seguridad jurídica, lo cual implica garantías } \\
\text { constitucionales a la propiedad y a la libertad económica, } \\
\text { así como las reglas de juego estables que no sean } \\
\text { afectadas por impulsos populistas o socialistas. } \\
\text { Los propósitos de las reformas son por un lado, fortalecer } \\
\text { el sistema penal por medio del aumento de penas y de la } \\
\text { disminución de la impunidad, por otro lado, eliminar el } \\
\text { activismo judicial en materia constitucional, con el } \\
\text { propósito de favorecer la seguridad jurídica de los } \\
\text { derechos de propiedad. (García, et al, 2006: } 69-70 \text { ) }\end{array}$ \\
\hline Teoría de la agencia & $\begin{array}{l}\text { La aplicación de los fundamentos de la teoría de la } \\
\text { agencia a la toma de decisiones y a la implementación de } \\
\text { las políticas, identifica como fundamento de las acciones, } \\
\text { públicas o privadas, individuales o colectivas, el mismo } \\
\text { principio maximizador. La cuestión central pasa a ser la } \\
\text { modalidad de contrato que se establece entre el principal } \\
\text { (los electores/o agencia gubernamental que actúe en su }\end{array}$ \\
\hline
\end{tabular}




\begin{tabular}{|c|c|}
\hline $\begin{array}{l}\text { CORRIENTES } \\
\text { TEÓRICAS }\end{array}$ & $\begin{array}{l}\text { REFORMA JUDICIAL: CONTENIDOS, ACCIONES Y } \\
\text { PROPUESTAS }\end{array}$ \\
\hline Teoría de la agencia & $\begin{array}{l}\text { nombre) y el agente (el ejecutor del gobierno o del } \\
\text { mercado), y los costos para hacer que sean cumplidas } \\
\text { sus cláusulas. En el campo de las actividades públicas } \\
\text { tienen consideración especial las dificultades que los } \\
\text { interesados últimos, o sea los electores, tienen para } \\
\text { ejercer el papel de principal, sea directamente o a través } \\
\text { de una cadena de relaciones del tipo "principal-agente". } \\
\text { Esa cadena involucra a políticos, burócratas y hasta } \\
\text { proveedores privados de servicios, ya que el interés de } \\
\text { los ciudadanos no es necesariamente coincidente con los } \\
\text { intereses de cualquiera de los agentes mencionados. } \\
\text { (Fleury, 2003:87) Como ejemplo, los sistemas de } \\
\text { servicios sociales -educación, salud, seguridad social- } \\
\text { deben ser reformados a partir de la diferenciación de las } \\
\text { funciones de los que financian, contratan, proveen } \\
\text { servicios, controlan, etc. Cada agencia debe encargarse } \\
\text { de funciones distintas ejerciendo, en relación con las } \\
\text { demás, el papel de principal o de agente, pero nunca los } \\
\text { dos juntos. (Fleury, 1999: 74) }\end{array}$ \\
\hline
\end{tabular}

\section{Múltiples miradas}

Una tendencia muy clara en los noventas fue la discusión sobre gobernabilidad y sobre la protección y desarrollo de la ciudadanía democrática en América Latina, dando lugar a una nueva y atenta mirada sobre la importancia de la administración de justicia dentro del conjunto del sistema político. Los procesos de transición a la democracia en la región han estado indudablemente ligados a un examen crítico del papel jugado por los jueces durante las dictaduras. La transición es el foco de estudios dirigidos hacia una segunda transición política en la cual es clave la afirmación de la dimensión republicana de la democracia. Estos estudios se 


\section{REVISTA VIRTUAL VIA INVENIENDI ET IUDICANDI \\ "CAMINO DEL HALLAZGO Y DEL JUICIO" \\ http://viei.usta.edu.co/ E-MAIL: revistainveniendi@usantotomas.edu.co}

hicieron desde enfoques disímiles que no tienen una continuidad y en Colombia en particular se carece de estudios que profundicen esta perspectiva entre otras razones por las características del régimen político colombiano.

Se destacan algunos trabajos publicados en este sentido como el de Justicia y democracia en Colombia: ¿en entredicho? que da cuenta de aspectos como la independencia de la judicatura en Colombia (Gómez Albarello: 1996) la situación de precaria autonomía de los jueces ordinarios, la justicia militar, el acceso a la justicia donde la sociedad colombiana enfrenta con dramatismo el hecho de que la oferta de justicia no ha sido monopolizada con éxito por el Estado, y por ello las guerrillas, los paramilitares y, de manera ilegal las fuerzas armadas, administran justicia a su modo.

El autor respecto de la tardía y difícil solución efectiva de los conflictos en el país afirma, que aunque es grave y que es una problemática de vieja data, sostiene que los nuevos mecanismos constitucionales lentamente han mejorado la situación como es el caso de la acción de tutela.

Los temas más recurrentes abordados son a saber: la independencia de la judicatura en Colombia, el acceso a la justicia (la oferta de mecanismos informales de resolución de conflictos, los nuevos mecanismos de protección de los derechos). 


\section{REVISTA VIRTUAL VIA INVENIENDI ET IUDICANDI \\ "CAMINO DEL HALLAZGO Y DEL JUICIO" \\ http://viei.usta.edu.co/E-MAIL: revistainveniendi@usantotomas.edu.co}

La judicialización de la política y la politización de la justicia: el papel político del control constitucional, la Corte Constitucional y el cambio de la relación entre el derecho y la política, la Corte Constitucional como nuevo poder real dentro del sistema político y el complicado escenario del activismo de la Corte.

En la globalización del Estado de derecho: el neoconstitucionalismo, el neoliberalismo y la transformación institucional en América Latina, César Rodríguez Garavito(2009) toma como base la globalización y expansión del estado derecho concentrándose en América Latina, y en un tipo específico de transformación institucional (la reforma judicial) que ha sido importante en las últimas dos décadas en nuestro país.

Frente a la proliferación de proyectos para el estado de derecho existe un activo debate académico que Rodríguez expresa como sigue:

Los estudios existentes tienen dos importantes limitaciones. En primer lugar, los análisis de los participantes en el campo y de los académicos están dominados por igual por los enfoques instrumentales que se concentran en la evaluación del éxito o el fracaso del programa (Trebilcock y Daniels 2008; IDB 1998; Shihata 1995; Domingo y Sieder 2001). Están guiados por preocupaciones como determinar cuáles son las mejores prácticas, evaluar cuáles son las condiciones para los trasplantes institucionales exitosos y, en 


\section{REVISTA VIRTUAL VIA INVENIENDI ET IUDICANDI \\ "CAMINO DEL HALLAZGO Y DEL JUICIO" \\ http://viei.usta.edu.co/ E-MAIL: revistainveniendi@usantotomas.edu.co}

tiempos más recientes, "empoderar" a las personas relevantes para que participen en los procesos de reforma (Dakolias 2008, 1996; Prillaman 2000). Aunque estos trabajos pueden ser útiles para la elaboración de políticas públicas, el enfoque instrumentalista es incapaz de capturar las luchas de poder que subyacen a los procesos de reforma judicial.

En esos enfoques está ausente el análisis sociológico del papel de los actores concretos en el campo del estado global de derecho y también las tensiones y vínculos entre ellos, es decir, entre agencias financiadoras transnacionales, gobiernos nacionales, abogados de las empresas y activistas de las ONG, por mencionar algunos. Lo que se requiere, por lo tanto, es un examen de cómo los actores en este campo han luchado por definir la teoría y la práctica del estado de derecho y de la reforma judicial. En el mismo sentido el autor menciona:

Una segunda carencia de las obras académicas es no haber captado la diversidad de concepciones sobre el (Estado de Derecho) ED y los proyectos políticos que están interviniendo en torno a él. De hecho, los estudios y las declaraciones de política pública tienden a eludir el problema de qué se quiere decir exactamente cuando se habla de ED y, en consecuencia, hacen que este concepto se convierta en un término multipropósito, que puede referirse a cualquier cosa, y que incluiría características institucionales tan variadas -y potencialmente contradictorias- como la independencia judicial, la responsabilidad pública por los actos políticos, el acceso a los tribunales, 


\section{REVISTA VIRTUAL VIA INVENIENDI ET IUDICANDI \\ "CAMINO DEL HALLAZGO Y DEL JUICIO" \\ http://viei.usta.edu.co/ E-MAIL: revistainveniendi@usantotomas.edu.co}

una burocracia competente, la seguridad jurídica y la protección de los derechos humanos. En un nivel analítico, esta imprecisión conceptual obscurece, cuando menos, la existencia de diferentes teorías y concepciones sobre el ED.

Agrega Rodríguez que el nivel sociológico, oculta las diferencias políticas y jurídicas entre proyectos políticos de reforma del estado de derecho, discrepantes entre sí, y también las luchas existentes entre los actores nacionales y transnacionales que promueven esos proyectos. Propone dos concepciones para abordar el estudio del tema, por un lado, las denominadas concepciones ligeras que privilegian la función estabilizadora del estado de derecho y que se concentran en la seguridad jurídica, es decir, en el estado de derecho weberiano como fuente de reglas de juego predecibles. Las concepciones ligeras incluyen tanto corrientes que son indiferentes al contenido de las leyes que proporcionan seguridad jurídica (y por lo tanto son compatibles con regímenes no democráticos) como otras que, a la vez que subrayan el orden público y la libertad económica, defienden los derechos civiles y políticos como límites al poder del Estado (Rodríguez 2009:16).

De otro lado se encuentran las concepciones "densas", que privilegian el aspecto del estado de derecho que otorga una mayor capacidad al ciudadano para realizar la vida que desea. Esta perspectiva -que se basa en una comprensión expansiva 


\section{REVISTA VIRTUAL VIA INVENIENDI ET IUDICANDI \\ "CAMINO DEL HALLAZGO Y DEL JUICIO"}

http://viei.usta.edu.co/E-MAIL: revistainveniendi@usantotomas.edu.co

de los derechos civiles, políticos y sociales- fue expresada en la conocida Declaración de Delhi, redactada por un colectivo de profesionales progresistas del derecho de todo el mundo, convocados en 1959 por la Comisión Internacional de Juristas. Según esa Declaración, "el estado de derecho... debería utilizarse no sólo para garantizar y promover los derechos civiles y políticos del individuo en una sociedad libre, sino también para establecer las condiciones sociales, económicas, educativas y culturales en las que sus aspiraciones legítimas y su dignidad pueden llegar a hacerse realidad" (Rodríguez 2009:16)

Después de dos décadas de inversión en la reforma judicial y de coexistencia del neoliberalismo y el neoconstitucionalismo, se pregunta el autor con razón ¿cuál ha sido el efecto sobre el campo del estado de derecho colombiano?

Destaca Rodríguez que el itinerario de la reforma institucional colombiana ha seguido un círculo completo. Al igual que en el momento fundacional de la Asamblea Constituyente de 1991, la convergencia de los proyectos neoliberal y neoconstitucional (esta vez bajo la égida de un neoliberalismo revisado o "arrepentido") constituye el consenso subyacente a la reforma del Estado de derecho, como ilustra la transformación del sistema de justicia penal.(Rodríguez, 2009: 70) 


\section{REVISTA VIRTUAL VIA INVENIENDI ET IUDICANDI \\ "CAMINO DEL HALLAZGO Y DEL JUICIO" \\ http://viei.usta.edu.co/ E-MAIL: revistainveniendi@usantotomas.edu.co}

\section{Fondo vs superficie: A guisa de cierre}

Un elemento común dentro de los textos mencionados, radica en la relación que plantean entre los elementos conceptuales y las orientaciones que para América Latina y para Colombia, han tenido los procesos de administración de justicia y reforma judicial. Sin embargo, resulta importante señalar, que no es éste el carácter del grueso grupo de literatura producida en el tema, quizá, debido a la tendencia mayoritaria de caracterizar el estado de la discusión pública, del proceso de reforma judicial o de la situación de la administración de justicia en el país sin comprometerse los autores, en estos análisis, con la fundamentación teórica que pudieran inspirar las mismas reformas que estudian, así como la corriente propia desde la que se posicionan para dicho análisis. Los aspectos mencionados, responden principalmente a las características de documentos sobre el tema entre los que se comprenden, informes institucionales de la rama judicial, compilaciones que sistematizan la experiencia de técnicos y expertos que han trabajado en el tema de la reforma en el país, memorias de congresos y eventos nacionales e internacionales que han versado sobre lo mismo, pero también, estudios provenientes de la academia en los que el sustrato teórico no es explícitamente identificado y abordado. En estos últimos recaen preguntas como ¿Cuál es el aporte para las ciencias sociales de estudios en el tema de reforma judicial que no discuten desde una orientación teórica específica? ¿Qué validez tienen los resultados de estudios e investigaciones realizadas sobre los que no se 


\section{REVISTA VIRTUAL VIA INVENIENIDI ET IUDICANDI \\ "CAMINO DEL HALLAZGO Y DEL JUICIO"}

http://viei.usta.edu.co/E-MAIL: revistainveniendi@usantotomas.edu.co

conoce claramente el enfoque teórico? ¿Cómo se da la cualificación de las ciencias sociales sin fortalecer el acumulado de discusión teórica? ¿Qué tipo de implicaciones metodológicas tiene el descentramiento teórico para la investigación científica?

Por otro lado interesa mencionar cómo en la literatura revisada que aborda explícitamente el componente teórico, lo que prima es un ejercicio expositivo y sintético de los hechos de reforma judicial desde una postura exógena al debate mismo de lo teórico, es decir, los autores exploran las fuentes teóricas que han dado cuerpo a las reformas judiciales en el país, en algunos casos presentan las controversias (suscitadas por otros) que se enlazan en el desarrollo de los postulados teóricos, pero no se enfrentan al debate con un enfoque desde el que discutan las otras corrientes o generen propuestas analíticas para futuras investigaciones. Sin embargo, este no es el caso de autores como García, Rodríguez y Uprimny que al hacer su estudio sobre reforma judicial, no sólo recogen los principales postulados y conceptos relacionados con el tema, sino que los discuten y critican, proponiendo a su vez, una propia y nueva corriente teórica desde la que justifican sus argumentos y generan estrategias y aportes al debate académico y al público sobre reforma judicial. 


\section{REVISTA VIRTUAL VIA INVENIENIDI ET IUDICANIDI}

"CAMINO DEL HALLAZGO Y DEL JUICIO"

http://viei.usta.edu.co/ E-MAIL: revistainveniendi@usantotomas.edu.co

\section{BIBLIOGRAFÍA}

FUENTES, Alfredo. (2005) "La Reforma en Colombia: Tendencias Recientes 1991-2003," En Luis Pásara, (comp.), En Busca de una Justicia Distinta. Experiencias de Reforma en América Latina, Justicia Viva, Lima, Perú (2004): 141 193.

GARCÍA CHOURIO, José. De la primera a la segunda generación de reformas del Estado en América Latina: giro ideológico y cambio conceptual. Cuadernos de Economía. Volumen XXII. No 38. Bogotá. 2003. Págs. 95-125

GARCÍA, Mauricio. (2006) "Reformismo introvertido. Las transformaciones de la justicia en Colombia durante los últimos treinta años." En LEAL B. Francisco. En la encrucijada Colombia en el siglo XXI" Grupo Editorial Norma Colección Virtral, Bogotá.

GÓMEZ ALBARELLO, Juan Gabriel. (1996) Justicia y democracia en Colombia ¿en entredicho?, Revista Análisis Político No 28 Instituto de Estudios Políticos y Relaciones Internacionales. UNAL

RODRÍGUEZ, Cesar. (2009) La Globalización del Estado de derecho. Ediciones Uniandes. Universidad de los Andes, Facultad de Derecho, Centro de Investigaciones Socio jurídico. 


\section{REVISTA VIRTUAL VIA INVENIENIDI ET IUDICANDI \\ "CAMINO DEL HALLAZGO Y DEL JUICIO"}

http://viei.usta.edu.co/ E-MAIL: revistainveniendi@usantotomas.edu.co

UPRIMNY, Rodrigo, RODRÍGUEZ, Cesar y GARCÍA, Mauricio.(2006) Justicia, democracia y violencia en Colombia: la evolución del sistema judicial en las últimas décadas. En: Mauricio García Villegas. Rodrigo Uprimny y César Rodríguez. ¿Justicia para todos? Sistema judicial, derechos sociales y democracia en Colombia. Bogotá: Norma.

UPRIMNY, Rodrigo. (2006) "Entre el protagonismo, la precariedad y las amenazas: las Paradojas de la judicatura." En Leal Buitrago, Francisco En la encrucijada Colombia en el siglo XXI" Grupo Editorial Norma Colección Virtral, Bogotá.

UPRIMNY, Rodrigo, RODRIGUEZ, Cesar y GARCIA, Mauricio.(2006) Justicia, democracia y violencia en Colombia: la evolución del sistema judicial en las últimas décadas. En: Mauricio García Villegas. Rodrigo Uprimny y César Rodríguez. ¿Justicia para todos? Sistema judicial, derechos sociales y democracia en Colombia. Bogotá: Norma 